International Mathematical Forum, Vol. 8, 2013, no. 27, 1337 - 1344

HIKARI Ltd, www.m-hikari.com

http://dx.doi.org/10.12988/imf.2013.3595

\title{
Coefficient Bounds for Certain Subclasses of Bi-univalent Functions
}

\author{
N. Magesh \\ Post-Graduate and Research Department of Mathematics \\ Government Arts College for Men \\ Krishnagiri 635001, Tamilnadu, India \\ nmagi_2000@yahoo.co.in \\ J. Yamini \\ Department of Mathematics, Govt First Grade College \\ Vijayanagar, Bangalore-560104, Karnattaka, India \\ yaminibalaji@gmail.com
}

Copyright (c) 2013 N. Magesh and J. Yamini. This is an open access article distributed under the Creative Commons Attribution License, which permits unrestricted use, distribution, and reproduction in any medium, provided the original work is properly cited.

Abstract. In this paper, we introduce and investigate two new subclasses of the function class $\Sigma$ of bi-univalent functions. Also, we find estimates of $\left|a_{2}\right|$ and $\left|a_{3}\right|$. Some related consequences of the results are also pointed out.

\section{Mathematics Subject Classification: 30C45}

Keywords: Analytic functions; Univalent functions; Bi-univalent functions; Bi-starlike and Bi-convex functions

\section{INTRODUCTION}

Let $\mathcal{A}$ denote the class of functions of the form

$$
f(z)=z+\sum_{n=2}^{\infty} a_{n} z^{n}
$$

which are analytic in the open unit disc $\mathbb{U}=\{z: z \in \mathbb{C}$ and $|z|<1\}$. Further, by $\mathcal{S}$ we shall denote the class of all functions in $\mathcal{A}$ which are univalent in $\mathbb{U}$. 
Some of the important and well-investigated subclasses of the univalent function class $\mathcal{S}$ include (for example) the class $\mathcal{S}^{*}(\alpha)$ of starlike functions of order $\alpha$ in $\mathbb{U}$ and the class $\mathcal{K}(\alpha)$ of convex functions of order $\alpha$ in $\mathbb{U}$. By definition, we have

$$
\mathcal{S}^{*}(\alpha):=\left\{f: f \in \mathcal{S} \text { and } \Re\left(\frac{z f^{\prime}(z)}{f(z)}\right)>\alpha ; z \in U ; 0 \leq \alpha<1\right\}
$$

and

$$
\mathcal{K}(\alpha):=\left\{f: f \in \mathcal{S} \text { and } \Re\left(1+\frac{z f^{\prime \prime}(z)}{f^{\prime}(z)}\right)>\alpha ; z \in U ; \quad 0 \leq \alpha<1\right\} .
$$

It readily follows from the definitions (1.1.2) and (1.1.3) that

$$
f \in \mathcal{K}(\alpha) \Longleftrightarrow z f^{\prime} \in \mathcal{S}^{*}(\alpha) \text {. }
$$

It is well known that every function $f \in \mathcal{S}$ has an inverse $f^{-1}$, defined by $f^{-1}(f(z))=z, z \in \mathbb{U}$ and $f\left(f^{-1}(w)\right)=w,|w|<r_{0}(f) ; r_{0}(f) \geq \frac{1}{4}$, where

$$
f^{-1}(w)=w-a_{2} w^{2}+\left(2 a_{2}^{2}-a_{3}\right) w^{3}-\left(5 a_{2}^{3}-5 a_{2} a_{3}+a_{4}\right) w^{4}+\ldots
$$

A function $f \in \mathcal{A}$ is said to be bi-univalent in $\mathbb{U}$ if both $f(z)$ and $f^{-1}(z)$ are univalent in $\mathbb{U}$. Let $\Sigma$ denote the class of bi-univalent functions in $\mathbb{U}$ given by (1.1.1). Examples of functions in the class $\Sigma$ are $\frac{z}{1-z},-\log (1-z), \frac{1}{2} \log \left(\frac{1+z}{1-z}\right)$ and so on. However, the familiar Koebe function is not a member of $\Sigma$. Other common examples of functions in $\mathcal{S}$ such as $z-\frac{z^{2}}{2}$ and $\frac{z}{1-z^{2}}$ are also not members of $\Sigma$ (see $[5,12])$.

In 1967, Lewin [7] investigated the bi-univalent function class $\Sigma$ and showed that $\left|a_{2}\right|<1.51$. Subsequently, Brannan and Clunie [2] conjectured that $\left|a_{2}\right| \leq \sqrt{2}$. Netanyahu [10], on the other hand, showed that $\max _{f \in \Sigma}\left|a_{2}\right|=\frac{4}{3}$. The coefficient estimate problem for each of the following Taylor-Maclaurin coefficients $\left|a_{n}\right|$ for $n \in \mathbb{N} \backslash\{1,2\} ; \mathbb{N}:=\{1,2,3, \ldots\}$ is presumably still an open problem.

A function $f \in \mathcal{A}$ is in the class $\mathcal{S}_{\Sigma}^{\alpha}$ of strongly bi-starlike of order $\alpha(0<$ $\alpha \leq 1$ ), if each of the following condition is satisfied:

$f \in \Sigma,\left|\arg \left(\frac{z f^{\prime}(z)}{f(z)}\right)\right|<\frac{\alpha \pi}{2}$, and $\left|\arg \left(\frac{w g^{\prime}(w)}{g(w)}\right)\right|<\frac{\alpha \pi}{2}, z, w \in \mathbb{U} ; 0<\alpha \leq 1$,

where the function $g$ is given by

$$
g(w)=w-a_{2} w^{2}+\left(2 a_{2}^{2}-a_{3}\right) w^{3}-\left(5 a_{2}^{3}-5 a_{2} a_{3}+a_{4}\right) w^{4}+\ldots
$$

the extension of $f^{-1}$ to $\mathbb{U}$.

The classes $\mathcal{S}_{\Sigma}^{*}(\alpha)$ and $\mathcal{K}_{\Sigma}(\alpha)$ of bi-starlike functions of order $\alpha$ and biconvex functions of order $\alpha$, corresponding to the function classes $\mathcal{S}^{*}(\alpha)$ and $\mathcal{K}(\alpha)$ defined by (1.1.2) and (1.1.3), were also introduced analogously. For each of the function classes $\mathcal{S}_{\Sigma}^{*}(\alpha)$ and $\mathcal{K}_{\Sigma}(\alpha)$, Brannan and Taha [4] found non-sharp estimates on the first two Taylor-Maclaurin coefficients $\left|a_{2}\right|$ and $\left|a_{3}\right|$ (for details see [4, 14]). Following Brannan and Taha [4], Srivastava et al. [12] 
introduced certain subclass $\mathcal{H}_{\Sigma}^{\alpha}, 0<\alpha \leq 1$ of the bi-univalent functions class $\Sigma$, a function $f(z)$ given by (1.1.1) is said to be in the class $\mathcal{H}_{\Sigma}^{\alpha}, 0<\alpha \leq 1$, if the following conditions are satified:

$$
f \in \Sigma,\left|\arg \left(f^{\prime}(z)\right)\right|<\frac{\alpha \pi}{2}, \text { and }\left|\arg \left(g^{\prime}(w)\right)\right|<\frac{\alpha \pi}{2}, z, w \in \mathbb{U} ; 0<\alpha \leq 1,
$$

where the function $g$ is given

$$
g(w)=w-a_{2} w^{2}+\left(2 a_{2}^{2}-a_{3}\right) w^{3}-\left(5 a_{2}^{3}-5 a_{2} a_{3}+a_{4}\right) w^{4}+\ldots .
$$

Then later many researchers (see $[1,6,15,16]$ ) studied extensively the same class $\mathcal{H}_{\Sigma}^{\alpha}$, by different techniques and found the non-sharp estimates on the first two Taylor-Maclaurin coefficients $\left|a_{2}\right|$ and $\left|a_{3}\right|$. It is interest to note that the estimates were found are improved but not sharp. Further, Frasin and Aouf [5] extended the class $\mathcal{H}_{\Sigma}^{\alpha}$, and obtained the non-sharp bounds (see also $[9,13])$.

Motivated by the aforementioned works, we introduce the following subclasses of the function class $\Sigma$.

Definition 1.1. A function $f(z)$ given by (1.1.1) is said to be in the class $\mathcal{S}_{\Sigma}(\alpha, \lambda)$ if the following conditions are satisfied:

$f \in \Sigma, \quad\left|\arg \left(\frac{z f^{\prime}(z)+\left(2 \lambda^{2}-\lambda\right) z^{2} f^{\prime \prime}(z)}{4\left(\lambda-\lambda^{2}\right) z+\left(2 \lambda^{2}-\lambda\right) z f^{\prime}(z)+\left(2 \lambda^{2}-3 \lambda+1\right) f(z)}\right)\right|<\frac{\alpha \pi}{2}$

$$
(0<\alpha \leqq 1 ; 0 \leqq \lambda \leqq 1 ; z \in \mathbb{U})
$$

and

$$
\begin{gathered}
\left|\arg \left(\frac{w g^{\prime}(w)+\left(2 \lambda^{2}-\lambda\right) w^{2} g^{\prime \prime}(w)}{4\left(\lambda-\lambda^{2}\right) w+\left(2 \lambda^{2}-\lambda\right) w g^{\prime}(w)+\left(2 \lambda^{2}-3 \lambda+1\right) g(w)}\right)\right|<\frac{\alpha \pi}{2} \\
(0<\alpha \leqq 1 ; 0 \leqq \lambda \leqq 1 ; w \in \mathbb{U})
\end{gathered}
$$

where the function $g$ is given by 1.1.6.

We note that for $\lambda=\frac{1}{2}$, the class $\mathcal{S}_{\Sigma}(\alpha, \lambda)$ reduces to the class $\mathcal{H}_{\Sigma}^{\alpha}$ introduced and studied by Srivastava et al. [12]. Putting $\lambda=0$, the class $\mathcal{S}_{\Sigma}(\alpha, \lambda)$ reduces to the class of strongly bi-starlike functions of order $\alpha(0<\alpha \leqq 1)$ and denoted by $\mathcal{S}_{\Sigma}^{*}(\alpha)$.

Definition 1.2. A function $f(z)$ given by (1.1.1) is said to be in the class $\mathcal{M}_{\Sigma}(\beta, \lambda)$ if the following conditions are satisfied:

$$
f \in \Sigma, \quad \Re\left(\frac{z f^{\prime}(z)+\left(2 \lambda^{2}-\lambda\right) z^{2} f^{\prime \prime}(z)}{4\left(\lambda-\lambda^{2}\right) z+\left(2 \lambda^{2}-\lambda\right) z f^{\prime}(z)+\left(2 \lambda^{2}-3 \lambda+1\right) f(z)}\right)>\beta
$$

$$
(0 \leqq \beta<1 ; 0 \leqq \lambda \leqq 1 ; z \in \mathbb{U})
$$


and

$$
\begin{gathered}
\Re\left(\frac{w g^{\prime}(w)+\left(2 \lambda^{2}-\lambda\right) w^{2} g^{\prime \prime}(w)}{4\left(\lambda-\lambda^{2}\right) w+\left(2 \lambda^{2}-\lambda\right) w g^{\prime}(w)+\left(2 \lambda^{2}-3 \lambda+1\right) g(w)}\right)>\beta \\
(0 \leqq \beta<1 ; 0 \leqq \lambda \leqq 1 ; w \in \mathbb{U}),
\end{gathered}
$$

where the function $g$ is given by (1.1.6).

It is interesting to note that, for $\lambda=\frac{1}{2}$ the class $\mathcal{M}_{\Sigma}(\beta, \lambda)$ reduces to the class $\mathcal{H}_{\Sigma}^{\beta}$ introduced and studied by Srivastava et al. [12]. Putting $\lambda=0$, the class $\mathcal{M}_{\Sigma}(\beta, \lambda)$ reduces to the class of bi-starlike functions of order $\beta(0<\beta \leqq 1)$ and denoted by $\mathcal{S}_{\Sigma}(\beta)$. When $\lambda=1$, the class $\mathcal{K}_{\Sigma}(\beta, \lambda)$ reduces to the class of bi-convex functions of order $\beta(0<\beta \leqq 1)$ and denoted by $\mathcal{K}_{\Sigma}(\beta)$.

The object of the present paper is to find estimates on the coefficients $\left|a_{2}\right|$ and $\left|a_{3}\right|$ for functions in the above-defined subclasses $\mathcal{S}_{\Sigma}(\alpha, \lambda)$ and $\mathcal{M}_{\Sigma}(\alpha, \lambda)$ of the function class $\Sigma$.

In order to derive our main results, we shall need the following lemma.

Lemma 1.3. ([11]) If $h \in \mathcal{P}$, then $\left|c_{k}\right| \leqq 2$ for each $k$, where $\mathcal{P}$ is the family of all functions $h$, analytic in $\mathbb{U}$, for which

$$
\Re\{h(z)\}>0 \quad(z \in \mathbb{U}),
$$

where

$$
h(z)=1+c_{1} z+c_{2} z^{2}+\cdots \quad(z \in \mathbb{U}) .
$$

2. Coefficient Bounds for the Function Class $\mathcal{S}_{\Sigma}(\alpha, \lambda)$

We begin by finding the estimates on the coefficients $\left|a_{2}\right|$ and $\left|a_{3}\right|$ for functions in the class $\mathcal{S}_{\Sigma}(\alpha, \lambda)$.

Theorem 2.1. Let the function $f(z)$ given by (1.1.1) be in the following class:

$$
\mathcal{S}_{\Sigma}(\alpha, \lambda) \quad(0<\alpha \leqq 1 ; 0 \leqq \lambda \leqq 1) .
$$

Then

$$
\left|a_{2}\right| \leqq \frac{2 \alpha}{\sqrt{\alpha\left(1-2 \lambda+25 \lambda^{2}-44 \lambda^{3}+20 \lambda^{4}\right)+\left(1+3 \lambda-2 \lambda^{2}\right)^{2}}}
$$

and

$$
\left|a_{3}\right| \leqq \frac{\alpha}{1+2 \lambda^{2}}+\frac{4 \alpha^{2}}{\left(1+3 \lambda-2 \lambda^{2}\right)^{2}} .
$$

Proof. It follows from (1.1.7) and (1.1.8) that

$$
\frac{z f^{\prime}(z)+\left(2 \lambda^{2}-\lambda\right) z^{2} f^{\prime \prime}(z)}{4\left(\lambda-\lambda^{2}\right) z+\left(2 \lambda^{2}-\lambda\right) z f^{\prime}(z)+\left(2 \lambda^{2}-3 \lambda+1\right) f(z)}=[p(z)]^{\alpha}
$$

and

$$
\frac{w g^{\prime}(w)+\left(2 \lambda^{2}-\lambda\right) w^{2} g^{\prime \prime}(w)}{4\left(\lambda-\lambda^{2}\right) w+\left(2 \lambda^{2}-\lambda\right) w g^{\prime}(w)+\left(2 \lambda^{2}-3 \lambda+1\right) g(w)}=[q(w)]^{\alpha},
$$


where $p(z)$ and $q(w)$ in $\mathcal{P}$ and have the following forms:

$$
p(z)=1+p_{1} z+p_{2} z^{2}+\cdots
$$

and

$$
q(z)=1+q_{1} w+q_{2} w^{2}+\cdots,
$$

respectively. Now, equating the coefficients in (2.2.3) and (2.2.4), we get

$$
\left(1+3 \lambda-2 \lambda^{2}\right) a_{2}=\alpha p_{1},
$$

$$
\begin{gathered}
\left(12 \lambda^{4}-28 \lambda^{3}+11 \lambda^{2}+2 \lambda-1\right) a_{2}^{2}+\left(4 \lambda^{2}+2\right) a_{3}=\frac{1}{2}\left[\alpha(\alpha-1) p_{1}^{2}+2 \alpha p_{2}\right] \\
-\left(1+3 \lambda-2 \lambda^{2}\right) a_{2}=\alpha q_{1}
\end{gathered}
$$

and

$$
\left(12 \lambda^{4}-28 \lambda^{3}+19 \lambda^{2}+2 \lambda+3\right) a_{2}^{2}-\left(4 \lambda^{2}+2\right) a_{3}=\frac{1}{2}\left[\alpha(\alpha-1) q_{1}^{2}+2 \alpha q_{2}\right] .
$$

From (2.2.7) and (2.2.9), we get

$$
p_{1}=-q_{1}
$$

and

$$
2\left(1+3 \lambda-2 \lambda^{2}\right)^{2} a_{2}^{2}=\alpha^{2}\left(p_{1}^{2}+q_{1}^{2}\right) .
$$

From (2.2.8), (2.2.10) and (2.2.12), we obtain

$$
a_{2}^{2}=\frac{\alpha^{2}\left(p_{2}+q_{2}\right)}{\alpha\left(1-2 \lambda+25 \lambda^{2}-44 \lambda^{3}+20 \lambda^{4}\right)+\left(1+3 \lambda-2 \lambda^{2}\right)^{2}} .
$$

Applying Lemma 1.3 for the coefficients $p_{2}$ and $q_{2}$, we immediately have

$$
\left|a_{2}\right| \leq \frac{2 \alpha}{\sqrt{\alpha\left(1-2 \lambda+25 \lambda^{2}-44 \lambda^{3}+20 \lambda^{4}\right)+\left(1+3 \lambda-2 \lambda^{2}\right)^{2}}}
$$

This gives the bound on $\left|a_{2}\right|$ as asserted in (2.2.1).

Next, in order to find the bound on $\left|a_{3}\right|$, by subtracting (2.2.10) from (2.2.8), we get

$$
2\left(2+4 \lambda^{2}\right) a_{3}-\left(8 \lambda^{2}+4\right) a_{2}^{2}=\alpha\left(p_{2}-q_{2}\right)+\frac{\alpha(\alpha-1)}{2}\left(p_{1}^{2}-q_{1}^{2}\right) .
$$

It follows from $(2.2 .11),(2.2 .12)$ and $(2.2 .13)$ that

$$
a_{3}=\frac{\alpha\left(p_{2}-q_{2}\right)}{2\left(2+4 \lambda^{2}\right)}+\frac{\alpha^{2}\left(p_{1}^{2}+q_{1}^{2}\right)\left(3 \lambda^{2}+1\right)}{2\left(2 \lambda^{2}+1\right)\left(1+3 \lambda-2 \lambda^{2}\right)^{2}} .
$$

Applying Lemma 1.3 once again, we readily get

$$
\left|a_{3}\right| \leq \frac{\alpha}{1+2 \lambda^{2}}+\frac{4 \alpha^{2}}{\left(1+3 \lambda-2 \lambda^{2}\right)^{2}} .
$$


This completes the proof of Theorem 2.1.

In the following section we find the estimates on the coefficients $\left|a_{2}\right|$ and $\left|a_{3}\right|$ for functions in the class $\mathcal{M}_{\Sigma}(\beta, \lambda)$.

3. Coefficient bounds for the FUnCtion Class $\mathcal{M}_{\Sigma}(\beta, \lambda)$

Theorem 3.1. Let $f(z)$ given by (1.1.1) be in the class $\mathcal{M}_{\Sigma}(\beta, \lambda), 0 \leq \beta<1$ and $0 \leq \lambda<1$. Then

$$
\left|a_{2}\right| \leq \sqrt{\frac{2(1-\beta)}{12 \lambda^{4}-28 \lambda^{3}+15 \lambda^{2}+2 \lambda+1}}
$$

and

$$
\left|a_{3}\right| \leq \frac{2(1-\beta)}{12 \lambda^{4}-28 \lambda^{3}+15 \lambda^{2}+2 \lambda+1} .
$$

Proof. It follows from (1.1.9) and (1.1.10) that there exists $p, q \in \mathcal{P}$ such that

$$
\frac{z f^{\prime}(z)+\left(2 \lambda^{2}-\lambda\right) z^{2} f^{\prime \prime}(z)}{4\left(\lambda-\lambda^{2}\right) z+\left(2 \lambda^{2}-\lambda\right) z f^{\prime}(z)+\left(2 \lambda^{2}-3 \lambda+1\right) f(z)}=\beta+(1-\beta) p(z)
$$

and

$$
\frac{w g^{\prime}(w)+\left(2 \lambda^{2}-\lambda\right) w^{2} g^{\prime \prime}(w)}{4\left(\lambda-\lambda^{2}\right) w+\left(2 \lambda^{2}-\lambda\right) w g^{\prime}(w)+\left(2 \lambda^{2}-3 \lambda+1\right) g(w)}=\beta+(1-\beta) q(w),
$$

where $p(z)$ and $q(w)$ have the forms (2.2.5) and (2.2.6), respectively. Equating coefficients in (3.3.3) and (3.3.4), we get

$$
\begin{gathered}
\left(1+3 \lambda-2 \lambda^{2}\right) a_{2}=(1-\beta) p_{1} \\
\left(12 \lambda^{4}-28 \lambda^{3}+11 \lambda^{2}+2 \lambda-1\right) a_{2}^{2}+\left(2+4 \lambda^{2}\right) a_{3}=(1-\beta) p_{2} \\
-\left(1+3 \lambda-2 \lambda^{2}\right) a_{2}=(1-\beta) q_{1}
\end{gathered}
$$

and

$$
\left(12 \lambda^{4}-28 \lambda^{3}+19 \lambda^{2}+2 \lambda+3\right) a_{2}^{2}-\left(2+4 \lambda^{2}\right) a_{3}=(1-\beta) q_{2} .
$$

From (3.3.5) and (3.3.7), we get

$$
p_{1}=-q_{1}
$$

and

$$
2\left(1+3 \lambda-2 \lambda^{2}\right)^{2} a_{2}^{2}=(1-\beta)^{2}\left(p_{1}^{2}+q_{1}^{2}\right) .
$$

Also, from (3.3.6), (3.3.8) and (3.3.10), we obtain

$$
a_{2}^{2}=\frac{(1-\beta)\left(p_{2}+q_{2}\right)}{2\left(12 \lambda^{4}-28 \lambda^{3}+15 \lambda^{2}+2 \lambda+1\right)} .
$$


Applying Lemma 1.3 for the coefficients $p_{2}$ and $q_{2}$, we immediately have

$$
\left|a_{2}\right| \leq \sqrt{\frac{2(1-\beta)}{12 \lambda^{4}-28 \lambda^{3}+15 \lambda^{2}+2 \lambda+1}} .
$$

This gives the bound on $\left|a_{2}\right|$ as asserted in (3.3.1).

Next, in order to find the bound on $\left|a_{3}\right|$, by subtracting (3.3.8) from (3.3.6), we get

$$
4\left(1+2 \lambda^{2}\right) a_{3}-4\left(1+2 \lambda^{2}\right) a_{2}^{2}=(1-\beta)\left(p_{2}-q_{2}\right) .
$$

It follows from (3.3.9), (3.3.10) and (3.3.11) that

$$
4\left(1+2 \lambda^{2}\right) a_{3}=\frac{4\left(1+2 \lambda^{2}\right)(1-\beta)\left(p_{2}+q_{2}\right)}{12 \lambda^{4}-28 \lambda^{3}+15 \lambda^{2}+2 \lambda+1}+(1-\beta)\left(p_{2}-q_{2}\right) .
$$

Applying Lemma 1.3 once again, we readily get

$$
\left|a_{3}\right| \leq \frac{2(1-\beta)}{12 \lambda^{4}-28 \lambda^{3}+15 \lambda^{2}+2 \lambda+1} .
$$

This completes the proof of Theorem 3.1.

Remark 3.2. Taking $\lambda=0$ in Theorem 2.1 and 3.1, the estimates on the coefficients $\left|a_{2}\right|$ and $\left|a_{3}\right|$ are improvement of the estimates on the first two Taylor-Maclaurin coefficients obtained in [8]. Also, for the choice of $\lambda=\frac{1}{2}$, the results stated in Theorem 2.1 and Theorem 3.1 would improve bounds stated in $[12]$.

Acknowledgements: The work is supported by UGC, under the grant F.MRP3977/11 (MRP/UGC-SERO) of the first author.

\section{REFERENCES}

[1] R. M. Ali, S.K.Lee, V.Ravichandran, S.Supramanian, Coefficient estimates for biunivalent Ma-Minda starlike and convex functions, Appl. Math. Lett. 25 (2012), no. 3, $344-351$.

[2] D.A. Brannan and J.G. Clunie (Editors), Aspects of Contemporary Complex Analysis, Academic Press, London, 1980.

[3] D. A. Brannan, J. Clunie and W. E. Kirwan, Coefficient estimates for a class of star-like functions, Canad. J. Math. 22 (1970), 476-485.

[4] D. A. Brannan and T. S. Taha, On some classes of bi-univalent functions, Studia Univ. Babeş-Bolyai Math. 31 (2) (1986), 70-77.

[5] B. A. Frasin and M. K. Aouf, New subclasses of bi-univalent functions, Appl. Math. Lett. 24 (2011), 1569-1573.

[6] T. Hayami and S. Owa, Coefficient bounds for bi-univalent functions, Pan Amer. Math. J. 22 (4) (2012), 15-26.

[7] M. Lewin, On a coefficient problem for bi-univalent functions, Proc. Amer. Math. Soc. 18 (1967), 63-68.

[8] X.-F. Li and A.-P. Wang, Two new subclasses of bi-univalent functions, Internat. Math. Forum 7 (2012), 1495-1504.

[9] G.Murugusundaramoorthy, N.Magesh and V.Prameela, Coefficient bounds for certain subclasses of bi-univalent functions, Abstract and Applied Analysis, Volume 2013, Article ID 573017, 3 pages. 
[10] E. Netanyahu, The minimal distance of the image boundary from the origin and the second coefficient of a univalent function in $z<1$, Arch. Rational Mech. Anal. 32 (1969), $100-112$.

[11] C. Pommerenke, Univalent Functions, Vandenhoeck \& Ruprecht, Göttingen, 1975.

[12] H. M. Srivastava, A. K. Mishra and P. Gochhayat, Certain subclasses of analytic and bi-univalent functions, Appl. Math. Lett. 23 (2010), 1188-1192.

[13] H.M.Srivastava, G. Murugusundaramoorthy and N. Magesh, On certain subclasses of bi-univalent functions associated with hohlov operator, Global Journal of Mathematical Analysis, 1 (2) (2013) 67-73.

[14] T. S. Taha, Topics in Univalent Function Theory, Ph.D. Thesis, University of London, 1981.

[15] Q.-H. Xu, Y.-C. Gui and H. M. Srivastava, Coefficient estimates for a certain subclass of analytic and bi-univalent functions, Appl. Math. Lett. 25 (2012), 990-994.

[16] Q.-H. Xu, H.-G. Xiao and H. M. Srivastava, A certain general subclass of analytic and bi-univalent functions and associated coefficient estimate problems, Appl. Math. Comput. 218 (2012), 11461-11465.

Received: May 4, 2013 